\title{
Serum Prognostic Biomarkers in Head and Neck Cancer Patients
}

\author{
Ho-Sheng Lin, MD, Fauzia Siddiq, PhD, Harvinder S. Talwar, PhD, Wei Chen, PhD, Calin \\ Voichita, PhD, Sorin Draghici, PhD, Gerald Jeyapalan, MD, Madhumita Chatterjee, PhD, \\ Andrew Fribley, PhD, George H. Yoo, MD, Seema Sethi, MD, Harold Kim, MD, Ammar \\ Sukari, MD, Adam J. Folbe, MD, and Michael A. Tainsky, PhD \\ Department of Surgery (H.-S.L.), John D. Dingell VA Medical Center, Detroit, Michigan; \\ Department of Otolaryngology-Head and Neck Surgery (H.-S.L., F.S., H.S.T., G.J., A.F., G.H.Y., \\ A.J.F.), Biostatistics Core (W.C.), Bioinformatics Core (C.V., S.D.), Program in Molecular Biology \\ and Genetics (M.C., M.A.T.), Developmental Therapeutics Program (A.F.), Department of \\ Pathology (S.S.),, Barbara Ann Karmanos Cancer Institute/Wayne State University and \\ Department of Oncology (M.A.T.), Barbara Ann Karmanos Cancer Institute/Wayne State \\ University, Detroit, Michigan; Department of Computer Science (C.V., S.D.), Department of \\ Radiation Oncology (H.K.), and Barbara Ann Karmanos Cancer Institute/Wayne State University; \\ and Department of Medicine (A.S.), Wayne State University, Detroit, Michigan; Carmen and Ann \\ Adams Department of Pediatrics, Division of Hematology Oncology (A.F.), Children's Hospital of \\ Michigan, Detroit, Michigan, U.S.A
}

\section{Abstract \\ Objectives/Hypothesis-A reliable estimate of survival is important as it may impact treatment choice. The objective of this study is to identify serum autoantibody biomarkers that can be used to improve prognostication for patients affected with head and neck squamous cell carcinoma (HNSCC). \\ Study Design—Prospective cohort study. \\ Methods-A panel of 130 serum biomarkers, previously selected for cancer detection using microarray-based serological profiling and specialized bioinformatics, were evaluated for their potential as prognostic biomarkers in a cohort of 119 HNSCC patients followed for up to 12.7 years. A biomarker was considered positive if its reactivity to the particular patient's serum was greater than one standard deviation above the mean reactivity to sera from the other 118 patients, using a leave-one-out cross-validation model. Survival curves were estimated according to the Kaplan-Meier method, and statistically significant differences in survival were examined using the log rank test. Independent prognostic biomarkers were identified following analysis using multivariate Cox proportional hazards models.}

(C) 2013 The American Laryngological, Rhinological and Otological Society, Inc.

Send correspondence to Ho-Sheng Lin, MD, Department of Otolaryngology/Head and Neck Surgery, Wayne State University, 5 East University Health Center, 4201 St. Antoine, Detroit, MI 48201. hlin@med.wayne.edu.

Level of Evidence: NA

Additional supporting information may be found in the online version of this article.

The authors have no other funding, financial relationships, or conflicts of interest to disclose. 
Results-Poor overall survival was associated with African Americans (hazard ratio [HR] for death $=2.61 ; 95 \%$ confidence interval $[\mathrm{CI}]: 1.58-4.33 ; P=.000)$, advanced stage $(\mathrm{HR}=2.79 ; 95 \%$ CI: $1.40-5.57 ; P=.004$ ), and recurrent disease (HR $=6.66 ; 95 \%$ CI: $2.54-17.44 ; P=.000$ ). On multivariable Cox analysis adjusted for covariates (race and stage), six of the 130 markers evaluated were found to be independent prognosticators of overall survival.

Conclusions-The results shown here are promising and demonstrate the potential use of serum biomarkers for prognostication in HNSCC patients. Further clinical trials to include larger samples of patients across multiple centers may be warranted.

\section{Keywords}

Head and neck squamous cell carcinoma; prognostic biomarkers; prognosis; proteomic

\section{INTRODUCTION}

Head and neck squamous cell carcinoma (HNSCC) is a devastating disease that afflicts approximately 49,260 Americans per year. ${ }^{1}$ Worldwide, HNSCC is the sixth most common malignancy, with an incidence rate of 644,000 new cases a year. ${ }^{2}$ Despite progress in diagnostic and treatment modalities in the past 30 years, long-term survival for patients affected by HNSCC has not significantly improved. ${ }^{3}$ In the United States, approximately 16,320 Americans will die from this disease each year. ${ }^{1}$

A reliable estimate of survival is important in clinical practice not only for patient counseling, but it may also impact the treating physicians' choice of therapy. Current available prognostic factors, such as tumor-node-metastasis (TNM) staging, provide only rough estimation of overall survival. ${ }^{4}$ Despite this imprecision, a major determinant of the aggressiveness of recommended treatment (single modality vs. multimodality) is often based on this TNM staging system. Other prognostic factors, such as surgical margin status, perineural and angiolymphatic invasion, number of lymph nodes involved, and extracapsular spread, have been shown to be helpful in guiding the aggressiveness of postoperative adjuvant therapy (radiation alone vs. chemoradiation) ${ }^{5,6}$ However, in this era of organ preservation, tissue specimens needed to assess these histopathologic factors is often not readily available. Recently, human papillomavirus (HPV) has received a significant amount of attention as a potential prognostic marker of improved outcome. ${ }^{7}$ Because of this prognostic information, several studies are investigating approaches to decrease the aggressiveness of treatment in patients with HPV-associated HNSCC to reduce treatment toxicity. ${ }^{8-10}$ The prognostic significance of other markers, such as p53 and epidermal growth factor receptor, are less clear, with some studies demonstrating an association with worse survival, ${ }^{11,12}$ whereas others showed no correlation. ${ }^{13,14}$

Autoantibodies against cancer-specific antigens have been identified in cancers of the colon, ${ }^{15}$ breast, ${ }^{16}$ lung, ${ }^{17}$ ovary, ${ }^{18}$ prostate,${ }^{19}$ and head and neck. ${ }^{20}$ The immune response with antibody production can be elicited due to the overexpression of cellular proteins ${ }^{21}$ such as HER-2/neu, ${ }^{22}$ the expression of mutated forms of cellular protein such as mutated $\mathrm{p} 53,{ }^{23}$ or the aberrant expression of tissue-restricted gene products such as cancer-testis antigens ${ }^{24}$ by cancer cells. Because these autoantibodies are raised against these specific 
antigens from the cancer cells, the detection of these antibodies in patients' sera can be exploited for cancer diagnosis and prognosis in these patients. Thus, the use of immune response as a biosensor through a serum-based assay holds great potential as an ideal diagnostic and prognostic tool.

A panel of 130 serum markers was previously selected for cancer detection using microarray-based serological profiling and specialized bioinformatics. This panel of markers was able to detect cancer with an accuracy of $84.9 \%$ (sensitivity of $79.8 \%$ and specificity of $90.1 \%$ ) based on its reactivity profile with serum antibodies in patients. ${ }^{25}$ Although not originally selected or intended as prognostic markers, six of these 130 biomarkers were identified as potentially useful prognostic biomarkers in a cohort of 119 HNSCC patients followed for up to 12.7 years in this study.

\section{MATERIALS AND METHODS}

\section{Serum Samples}

Blood samples from HNSCC patients, recruited from the otolaryngology-head and neck surgery clinic population, were obtained after informed consent. All enrolled HNSCC patients have cancer confirmed on pathology. Ten milliliters of peripheral blood were collected into red-top Vacutainers (Becton, Dicknson and Co., Franklin Lakes, NJ) without anticoagulant using standardized phlebotomy procedures. These samples were allowed to clot for 20 to 30 minutes at room temperature and then centrifuged at $2,500 \mathrm{rpm}$ at $4^{\circ} \mathrm{C}$ for 15 minutes. The supernatants or sera were immediately aliquoted and stored in $\mathrm{a}-70^{\circ} \mathrm{C}$ freezer. This study was conducted under protocols approved by the Human Investigation Committee (HIC \#121802MP4E).

\section{Serum Biomarkers}

A panel of 130 serum markers was previously selected for cancer detection using microarray-based serological profiling and specialized bioinformatics. ${ }^{25}$ This panel of markers was able to detect cancer with an accuracy of $84.9 \%$ based on its reactivity profile with serum antibodies in patients. ${ }^{25}$ Although not originally selected or intended as prognostic markers, these 130 markers were evaluated for their potential as prognostic biomarkers in this study.

\section{Protein Microarray Immunoreaction}

A total of 1,021 clones, including the 130 markers described above, were printed in replicates of five onto FAST slides (Schleicher \& Schuell/Whatman, Keene, NH) using a robotic microarrayer Prosys 5510TL (Cartesian Technologies, Irvine, CA) with 32 MicroSpotting Pins (TeleChem, Sunnyvale, CA). Protein microarrays were incubated with primary antibodies consisting of human serum at a dilution of 1:300 in phosphate-buffered saline, mouse anti-T7 capsid antibodies $(0.15 \mu \mathrm{g} / \mathrm{mL})$ (EMD Biosciences, Madison, WI), and BL21 Escherichia coli cell lysates $(5 \mu \mathrm{g} / \mathrm{mL})$. The microarrays were then washed and incubated with AlexaFluor647 (red fluorescent dye)-labeled goat anti-human immunoglobulin (Ig) $\mathrm{G}$ antibodies $(1 \mu \mathrm{g} / \mathrm{mL})$ and AlexaFluor532 (green fluorescent dye)- 
labeled goat anti-mouse IgG antibodies $(0.05 \mu \mathrm{g} / \mathrm{mL})$ (Molecular Probes, Eugene, OR) for 1 hour in the dark. Finally, the microarrays were washed and air dried.

\section{Data Acquisition and Preprocessing}

Following immunoreaction, the microarrays were scanned using the GenePix 4100A scanner (Axon Laboratories, Palo Alto, CA) using $635 \mathrm{~nm}$ and $532 \mathrm{~nm}$ lasers to produce a red (AlexaFluor647) and green (AlexaFluor532) composite image. Using the ImaGene 6.0 (Biodiscovery, Inc., Marina Del Rey, CA) image analysis software, the binding of each of the cancer-specific peptides with IgGs in each serum was then analyzed and expressed as a ratio of red-to-green fluorescent intensities. The microarray data were further read into the $\mathrm{R}$ environment (v2.3.0; R Foundation for Statistical Computing, Vienna, Austria) ${ }^{26}$ and processed by a sequence of transformations including background correction, omission of poor quality spots, base 2 log-transformation, LOESS (LOcal regrESSion)-based global normalization, and combining spot replicates into a single value for each marker. Specialized bioconductor (www.bio-conductor.org) libraries such as limma ${ }^{27}$ were used to this end.

\section{Sequencing of Phage cDNA Clones}

Individual phage clones were polymerase chain reaction amplified using forward primer 5'GTTCTATCCGCAACGTTAT GG-3' and reverse primer 5'GGAGGAAAGTCGTTTTTTGGGG-3' and sequenced using forward primer by Wayne State University Sequencing Core Facility.

\section{Statistical Analysis}

A biomarker was considered positive if its reactivity to the particular patient's serum was greater than one standard deviation above the mean reactivity to sera from the other 118 patients, using leave-one-out cross-validation method. Association between biomarker immunoreactivity and conventional prognostic factors was evaluated using Pearson $\chi^{2}$ tests. Survival was defined as the time from study entry and diagnosis to death. Survival curves, based on whether the biomarker is reactive or nonreactive to the sera from these 119 patients, were estimated according to the Kaplan-Meier method, and statistically significant differences in survival were examined using the log-rank test. Significant prognostic markers identified by log-rank test were then subjected to further analysis using the multivariable Cox proportional hazards models adjusted for conventional prognostic factors to identify independent prognostic markers. Proportional hazard assumptions were checked.

\section{RESULTS}

\section{Patient Characteristics}

A total of 119 HNSCC patients were followed for a period ranging from 1.2 months to 152.9 months, with a median of 30.5 months. The follow-up period is defined as from time of diagnosis to time of death or last follow-up. The age at diagnosis ranged from 26 to 82 years, with median of 59 years. The primary tumor site was unknown in nine patients (7.6\%), esophagus in three (2.5\%), glottis in $21(17.6 \%)$, supraglottis in $18(15.1 \%)$, oral cavity in $25(21.0 \%)$, nasopharynx in five (4.2\%), oropharynx in $26(21.8 \%)$, and 
hypopharynx in $12(10.1 \%)$. There were $22(18.5 \%)$ early-stage, 88 (73.9\%) late-stage, and nine $(7.6 \%)$ recurrent-stage patients. A majority of the patients were African American $(57.1 \%)$ and male $(79.02 \%)$. Treatment consisted of chemoradiation in 50 patients $(42.0 \%)$, surgery followed by chemoradiation in 47 (39.5\%), surgery alone in $11(9.2 \%)$, and radiation alone in $11(9.2 \%)$ (Table I).

\section{Overall Survival Based on Immunoreactivity of Markers}

Conventional prognostic factors, such as race and staging (early primary vs. late primary vs. recurrent), were found to correlate with overall survival in this study population (Fig. 1). Poor overall survival was associated with African American (hazard ratio for death, 2.61; 95\% confidence interval $[\mathrm{CI}]: 1.58-4.33 ; P=.000$ ), advanced stage (hazard ratio, $2.79 ; 95 \%$ CI: $1.40-5.57 ; P=.004$ ), and recurrent disease (hazard ratio, 6.66; 95\% CI: 2.54-17.44; $P=$. 000) (Table I).

Survival curves, based on whether the biomarker was reactive or nonreactive to the sera from these 119 patients, were estimated according to the Kaplan-Meier method. Proportional hazards models were used to assess the univariate prognostic significance of tumor markers on overall survival. Of the 130 biomarkers analyzed, increased immunoreactivity was associated with significantly worse survival (hazard ratios ranging from 1.76 to $2.82 ; P=$. 002 to .047) in 10 biomarkers (10_C3, 6_G11, 10_G12, 8_G3, 5_C3, 11_C3, 9_C2, 5_G4, 8_G8, and 9_H7) and associated with significantly better prognosis in two biomarkers (hazard ratio, $0.47 ; 95 \% \mathrm{CI}: 0.23-0.96 ; P=.038$ for $2 \_\mathrm{H} 3$ and hazard ratio, $0.47 ; 95 \% \mathrm{CI}$ : 0.24-0.96; $P=.037$ for 1_G8) (Table I). Using Pearson $\chi^{2}$ analysis, four of these 10 newly identified biomarkers correlated significantly with race (8_G3, 2_H3, 1_G8, and 9_H7), and two correlated significantly with stages (10_C3 and 11_C3). Biomarkers 8_G3 and 9_H7, which predict worse survival, were associated with African American. On the other hand, biomarkers 2_H3 and 1_G8 were associated with Caucasians, and they predicted better survival. In multivariable Cox analysis adjusted for covariates (race and stage), only six (10_C3,6_G11, 10_G12,9_C2, 5_G4, and 8_G8) of the 12 bio-markers were found to be independent prognostic markers of overall survival (Table II and Fig. 2). Thus, independent of stage or race, the presence of serum antibodies reactive to any of these six antigen biomarkers, as compared with the absence of reactive antibodies, increased the hazard ratio for death in these patients between two- to three-fold (Table II).

\section{Characterization of the Panel of Promising Prognostic Biomarkers}

The panel of 130 markers were sequenced and analyzed for homology to mRNA and genomic entries in the GenBank databases using BLASTn. The predicted amino acids inframe with the phage $\mathrm{T} 7$ gene 10 capsid protein were determined. Of the 12 biomarkers identified in this study, there was one clone (10_G12) that contained known gene products in the reading frame of the $\mathrm{T} 7$ gene 10 capsid proteins, and the remaining 11 clones contained peptides that were different from the original proteins coded by the inserted gene fragments. This occurred because the inserted gene fragments were out of frame with the open reading frame of the $\mathrm{T} 7 \mathrm{10B}$ gene, represented untranslated region of known genes, or contained sequences from unknown genes. It is likely that the recombinant gene products of these clones mimic some other natural antigens, and therefore can be termed 
mimotopes. ${ }^{18,28}$ It is also possible that some of these products may represent cancer antigens produced as result of altered reading frame or alternative splicing. ${ }^{29-31}$ BLASTp search of the SWISS-PROT database for homology to each in-frame mimotope revealed that many of these gene products mimic known cancer proteins and as such represented putative tumor antigens (Supplemental Table 1).

\section{DISCUSSION}

Reliable estimate of survival is important in clinical practice, as it may impact the choice of treatment to maximize effectiveness and minimize toxicity. Currently, TNM staging of the disease, which provides only a rough estimation of overall survival, is one of the most important factors that physicians take into consideration when recommending treatment options. Other prognostic factors, such as surgical margin status, extracapsular spread, number of positive lymph nodes, perineural and angiolymphatic invasion, can also help guide the aggressiveness of therapy. ${ }^{5,6}$ However, information with regard to these histopathologic factors is only available if surgical resection was undertaken. With the increasing use of nonsurgical treatment for head and neck cancer in many centers, surgical specimen is often not readily available for this histopathologic analysis. Because surgical resection was not part of the overall treatment plan in the majority of patients in this current study, these histopathologic factors were not available for majority of the patients in this study and were not included in the analysis as known prognostic factors. Further, because our center did not start collecting information on HPV status until about 2 years ago, we also did not have this information for analysis. This is clearly a limitation of this current study. Nonetheless, the advantage of a serum-based assay for prognostication is obvious. This prognostic information can be obtained without the need for expensive diagnostic imaging (required in most cases for accurate TNM staging) or the acquisition of a surgical specimen (required for histopathologic analysis). More importantly, the main utility of a serum-based prognostic assay may lie in the potential to improve the accuracy of prognostic estimation when combined with the currently available prognostic factors.

In this study, using Epitomics (Burlingame, CA) technology, ${ }^{32,33}$ based on a combination of high throughput antigen selection using microarray-based serological profiling and specialized bioinformatics, 12 biomarkers were identified as potentially promising candidates for prognostic applications in patients with HNSCC. After adjusting for known prognostic factors such as race and TNM staging, only six of the 12 biomarkers were found to be independent prognostic markers of overall survival. That is, the presence of serum antibodies reactive to any of these six biomarkers, as compared with the absence of such reactive antibodies, increased the patient's likelihood of death by two- to three-fold, independent of race or stage. Because the presence of advanced stage also increased the likelihood of death by two- to three-fold, each of these six markers actually has the same predictive power as the TNM staging system. Unlike the histopathologic variables mentioned earlier, the serum-based biomarkers are readily available irrespective of the type of treatment used and hold great potential as an ideal prognostic tool.

Of the six independent biomarkers identified in this study, five of them represented mimotopes, and only one (10_G12) represented a portion of a known protein C10. The 
functional significance of the gene (C12orf57) that codes for protein C10 is currently unknown. However, this gene is located at the CD4 locus of human chromosome 12p13, where it is clustered with genes that possess diverse expression patterns and functions, such as signal transduction, glycolysis, regulation of cell proliferation, and ubiquitin-dependent proteolysis. ${ }^{34,35}$ The other five biomarkers represented mimotopes, because they contained peptides that were different from the original proteins coded by the inserted gene fragments. However, BLASTp search of the SWISS-PROT database for homology to each in-frame mimotope revealed that many of these gene products contain regions of partial homology to known cancer proteins, such as serologically defined colon cancer antigen 33 variant, ovarian cancer-related tumor marker CA125, retinoblastoma-associated factor 600 , rhabdomyosarcoma antigen MU-RMS, and v-fos transformation effector protein.

We do acknowledge several limitations including a short follow-up and small number of patients in this study. These limitations will be addressed in future validation studies. Further, because of the large number of biomarkers used in the selection process, there is potential risk of identifying significant biomarkers due to chance (false discovery). Given the exploratory nature of this study, the markers identified here can serve as a springboard for future validation studies. More importantly, the results presented in this study indicate the potential of a new platform for head and neck cancer prognosis based on analysis of pattern of serum immunoreactivity against a panel of cancer antigens. This pattern of immunoreactivity was found to be highly reproducible. ${ }^{25}$ In addition, serum IgGs are extremely stable, which should minimize interlaboratory variations in the clinical diagnostic setting. Further, the potential to translate this approach into an assay system already widely available in clinical practice, enzyme linked immunosorbent assay, represents a major advantage of this technology. In addition to early detection and prognosis, these biomarkers may also have utility in post-treatment monitoring of HNSCC patients and may even provide new targets for therapeutic interventions or diagnostic imaging in future clinical trials. Because the host immune system can reveal molecular events (overexpression or mutation) critical to the genesis of HNSCC, this novel proteomics technology can also identify genes with mechanistic involvement in the etiology of the disease.

\section{CONCLUSION}

Using Epitomics technology 32,33 based on a combination of high throughput antigen selection using microarray-based serological profiling and specialized bioinformatics, a panel of 130 biomarkers were previously identified that can provide sufficient accuracy for a clinically relevant, serum-based cancer detection test based on the pattern of serum Ig binding. From this panel of 130 markers, six biomarkers were identified in this study as potentially promising prognostic bio-markers. The results shown here demonstrate that this technology is capable of identifying both diagnostic and prognostic antigen biomarkers. Further work will need to be done to identify a comprehensive set of independent biomarkers whose predictive power can be combined to facilitate prognostication of HNSCC to improve patient counseling as well as guide choice of therapy. 


\section{Supplementary Material}

Refer to Web version on PubMed Central for supplementary material.

\section{Acknowledgments}

The authors acknowledge the expert assistance of the Biostatistics, Bioinformatics, and the Applied Genomics Cores, Karmanos Cancer Institute, Wayne State University (P30CA022453).

H.-S.L. is supported by the VA Merit Review award (I01 BX007080) and set aside fund from EDRN (U01CA117478-01). S.D. is supported by NSF DBI-0234806, NIH 1R01HG003491, NSF CCF-0438970, 1R21 EB00990-01, and 1R01 NS045207-01. M.A.T. is supported by U01-CA117478-01.

\section{BIBLIOGRAPHY}

1. Jemal A, Siegel R, Xu J, Ward E. Cancer statistics, 2010. CA Cancer J Clin. 2010; 60:277-300. [PubMed: 20610543]

2. Parkin DM, Bray F, Ferlay J, Pisani P. Global cancer statistics, 2002. CA Cancer J Clin. 2005; 55:74-108. [PubMed: 15761078]

3. Clayman, GL.; Lippman, SM.; Laramore, GE.; Hong, WK. Head and NECK CANCER. In: Bast, RCKD.; Pollock, RE.; Weichselbaum, RR.; Holland, JF.; Frei, E., editors. Cancer Medicine. Hamilton, Ontario, Canada: B.C: Decker Inc; 2000. p. 1175-1220.

4. Vokes EE, Weichselbaum RR, Lippman SM, Hong WK. Head and neck cancer. N Engl J Med. 1993; 328:184-194. [PubMed: 8417385]

5. Bernier J, Domenge C, Ozsahin M, et al. Postoperative irradiation with or without concomitant chemotherapy for locally advanced head and neck cancer. N Engl J Med. 2004; 350:1945-1952. [PubMed: 15128894]

6. Cooper JS, Pajak TF, Forastiere AA, et al. Postoperative concurrent radiotherapy and chemotherapy for high-risk squamous-cell carcinoma of the head and neck. N Engl J Med. 2004; 350:1937-1944. [PubMed: 15128893]

7. Kong CS, Narasimhan B, Cao H, et al. The relationship between human papillomavirus status and other molecular prognostic markers in head and neck squamous cell carcinomas. Int J Radiat Oncol Biol Phys. 2009; 74:553-561. [PubMed: 19427557]

8. Shoushtari AN, Rahimi NP, Schlesinger DJ, Read PW. Survey on human papillomavirus/p16 screening use in oropharyngeal carcinoma patients in the United States. Cancer. 2010; 116:514 519. [PubMed: 19937955]

9. Hong AM, Dobbins TA, Lee CS, et al. Human papillomavirus predicts outcome in oropharyngeal cancer in patients treated primarily with surgery or radiation therapy. Br J Cancer. 2010; 103:1510 1517. [PubMed: 20959828]

10. Ang KK, Harris J, Wheeler R, et al. Human papillomavirus and survival of patients with oropharyngeal cancer. N Engl J Med. 2010; 363:24-35. [PubMed: 20530316]

11. Mineta H, Borg A, Dictor M, Wahlberg P, Akervall J, Wennerberg J. p53 mutation, but not p53 overexpression, correlates with survival in head and neck squamous cell carcinoma. Br J Cancer. 1998; 78:1084-1090. [PubMed: 9792155]

12. Psyrri A, Yu Z, Weinberger PM, et al. Quantitative determination of nuclear and cytoplasmic epidermal growth factor receptor expression in oropharyngeal squamous cell cancer by using automated quantitative analysis. Clin Cancer Res. 2005; 11:5856-5862. [PubMed: 16115926]

13. Bosch FX, Ritter D, Enders C, et al. Head and neck tumor sites differ in prevalence and spectrum of p53 alterations but these have limited prognostic value. Int J Cancer. 2004; 111:530-538. [PubMed: 15239130]

14. Aebersold DM, Froehlich SC, Jonczy M, et al. Expression of transforming growth factor-alpha, epidermal growth factor receptor and platelet-derived growth factors A and B in oropharyngeal cancers treated by curative radiation therapy. Radiother Oncol. 2002; 63:275-283. [PubMed: 12142091] 
15. Scanlan MJ, Chen YT, Williamson B, et al. Characterization of human colon cancer antigens recognized by autologous antibodies. Int J Cancer. 1998; 76:652-658. [PubMed: 9610721]

16. Disis ML, Calenoff E, McLaughlin G, et al. Existent T-cell and antibody immunity to HER-2/neu protein in patients with breast cancer. Cancer Res. 1994; 54:16-20. [PubMed: 7505195]

17. Diesinger I, Bauer C, Brass N, et al. Toward a more complete recognition of immunoreactive antigens in squamous cell lung carcinoma. Int J Cancer. 2002; 102:372-378. [PubMed: 12402307]

18. Chatterjee M, Mohapatra S, Ionan A, et al. Diagnostic markers of ovarian cancer by highthroughput antigen cloning and detection on arrays. Cancer Res. 2006; 66:1181-1190. [PubMed: 16424057]

19. Wang X, Yu J, Sreekumar A, et al. Autoantibody signatures in prostate cancer. N Engl J Med. 2005; 353:1224-1235. [PubMed: 16177248]

20. Carey TE, Kimmel KA, Schwartz DR, Richter DE, Baker SR, Krause CJ. Antibodies to human squamous cell carcinoma. Otolaryngol Head Neck Surg. 1983; 91:482-491. [PubMed: 6417595]

21. Ali-Fehmi R, Chatterjee M, Ionan A, et al. Analysis of the expression of human tumor antigens in ovarian cancer tissues. Cancer Biomark. 2010; 6:33-48. [PubMed: 20164540]

22. Disis ML, Bernhard H, Gralow JR, et al. Immunity to the HER-2/neu oncogenic protein. Ciba Found Symp. 1994; 187:198-207. discussion 207-111. [PubMed: 7540970]

23. Soussi T. p53 Antibodies in the sera of patients with various types of cancer: a review. Cancer Res. 2000; 60:1777-1788. [PubMed: 10766157]

24. Chen YT, Scanlan MJ, Sahin U, et al. A testicular antigen aberrantly expressed in human cancers detected by autologous antibody screening. Proc Natl Acad Sci U S A. 1997; 94:1914-1918. [PubMed: 9050879]

25. Lin HS, Talwar HS, Tarca AL, et al. Autoantibody approach for serum-based detection of head and neck cancer. Cancer Epidemiol Biomarkers Prev. 2007; 16:2396-2405. [PubMed: 18006929]

26. Akin DT, Lu MQ, Lu SJ, Kendall S, Rundegren J, Arnold RR. Bactericidal activity of different forms of lactoferrin. Adv Exp Med Biol. 1994; 357:61-70. [PubMed: 7762447]

27. Smyth, GK. Limma: linear models for microarray data. In: Gentleman, R.; Carey, V.; Dudoit, S.; Irizarry, R.; Huber, W., editors. Bioinformatics and Computational Biology Solutions Using R and Bioconductor. New York, NY: Springer; 2005. p. 397-420.

28. Geysen HM, Rodda SJ, Mason TJ. A priori delineation of a peptide which mimics a discontinuous antigenic determinant. Mol Immunol. 1986; 23:709-715. [PubMed: 2432410]

29. Wang RF, Parkhurst MR, Kawakami Y, Robbins PF, Rosenberg SA. Utilization of an alternative open reading frame of a normal gene in generating a novel human cancer antigen. J Exp Med. 1996; 183:1131-1140. [PubMed: 8642255]

30. David A, Mabjeesh N, Azar I, et al. Unusual alternative splicing within the human kallikrein genes KLK2 and KLK3 gives rise to novel prostate-specific proteins. J Biol Chem. 2002; 277:1808418090. [PubMed: 11834722]

31. Rimoldi D, Rubio-Godoy V, Dutoit V, et al. Efficient simultaneous presentation of NY-ESO-1/ LAGE-1 primary and nonprimary open reading frame-derived CTL epitopes in melanoma. $\mathrm{J}$ Immunol. 2000; 165:7253-7261. [PubMed: 11120859]

32. Chatterjee M, Ionan A, Draghici S, Tainsky MA. Epitomics: global profiling of immune response to disease using protein microarrays. OMICS. 2006; 10:499-506. [PubMed: 17233560]

33. Draghici S, Chatterjee M, Tainsky MA. Epitomics: serum screening for the early detection of cancer on microarrays using complex panels of tumor antigens. Expert Rev Mol Diagn. 2005; 5:735-743. [PubMed: 16149876]

34. Ansari-Lari MA, Oeltjen JC, Schwartz S, et al. Comparative sequence analysis of a gene-rich cluster at human chromosome 12 p13 and its syntenic region in mouse chromosome 6 . Genome Res. 1998; 8:29-40. [PubMed: 9445485]

35. Ansari-Lari MA, Muzny DM, Lu J, et al. A gene-rich cluster between the CD4 and triosephosphate isomerase genes at human chromosome 12p13. Genome Res. 1996; 6:314-326. [PubMed: 8723724] 

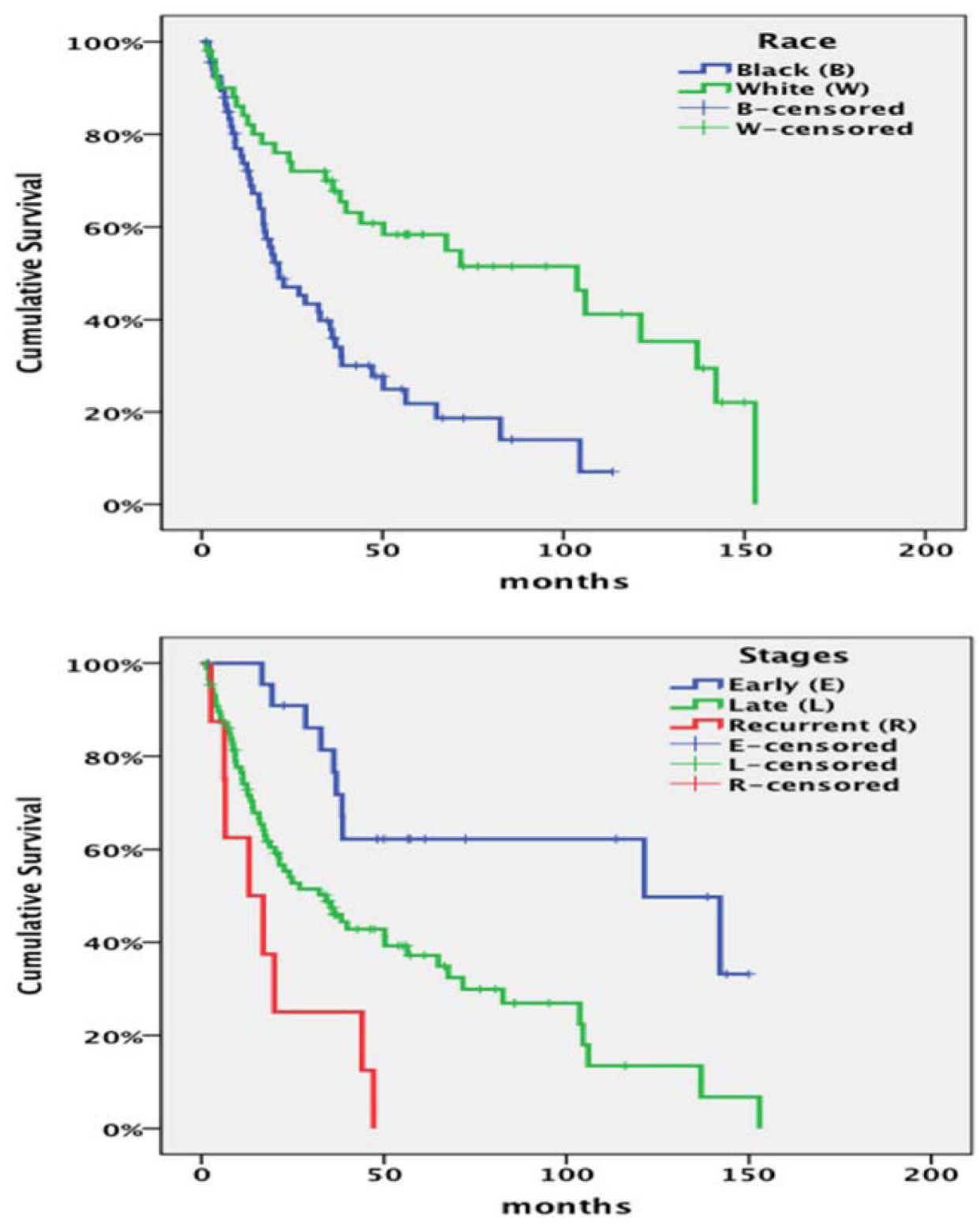

Fig. 1.

Kaplan-Meier survival curve as a function of race and staging (early primary versus late primary versus recurrent). Statistically significant worse overall survival was associated with African American ( $\mathrm{P}=.000)$ and advanced and recurrent stage ( $\mathrm{P}=.000)$. [Color figure can be viewed in the online issue, which is available at wileyonlinelibrary.com.] 

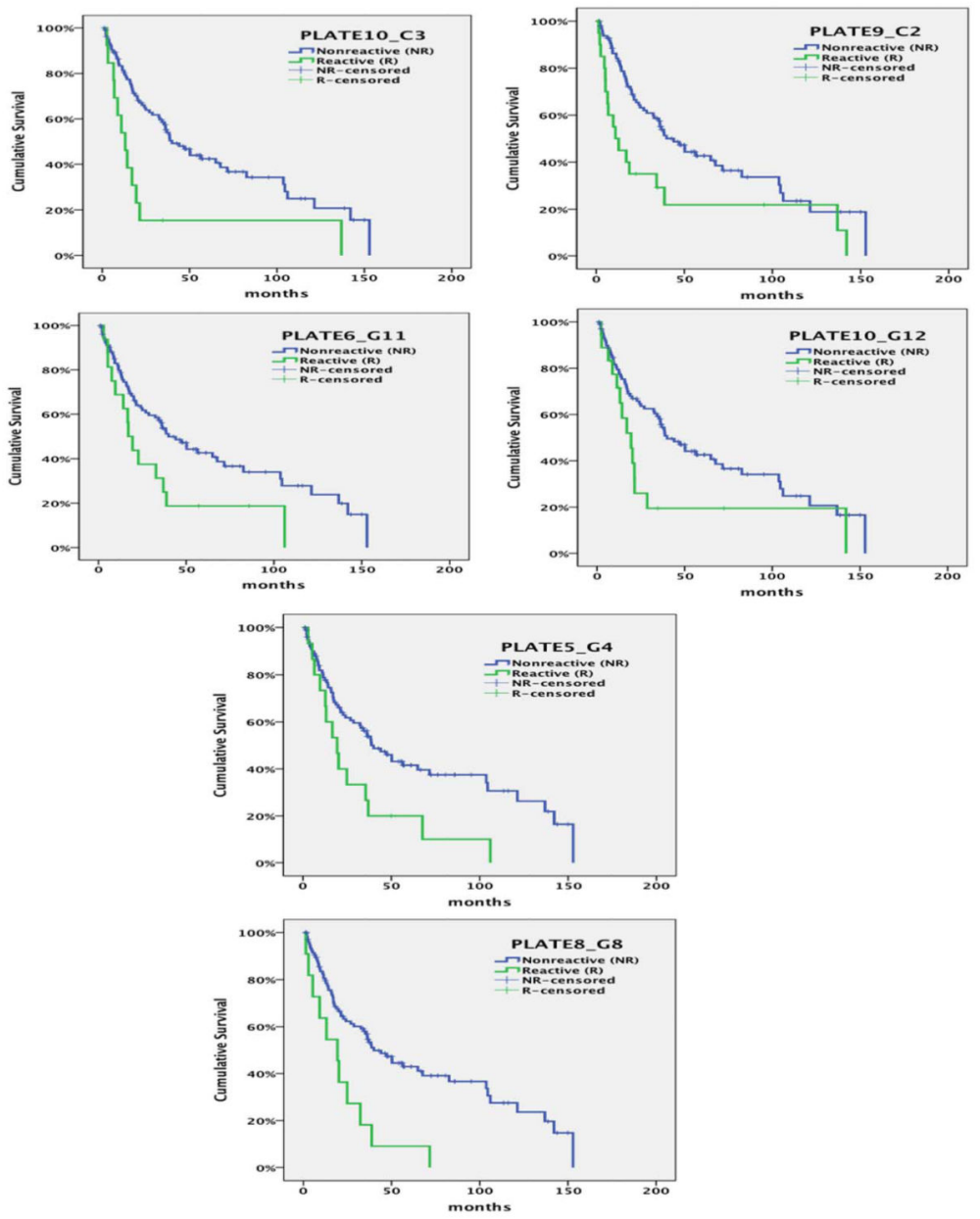

Fig. 2.

Kaplan-Meier survival curve for the six biomarkers (10_C3, 6_G11, 10_G12, 9_C2, 5_G4, and 8_G8) found to be independent prognostic markers of overall survival. Increased immunoreactivity to any of these six markers predicts worse survival outcome. Thus, independent of stage or race, the presence of serum antibodies reactive to any of these six biomarkers, as compared with the absence of reactive antibodies, increased the hazard ratio for death in these patients between two- to three-fold. [Color figure can be viewed in the online issue, which is available at wileyonlinelibrary.com.] 


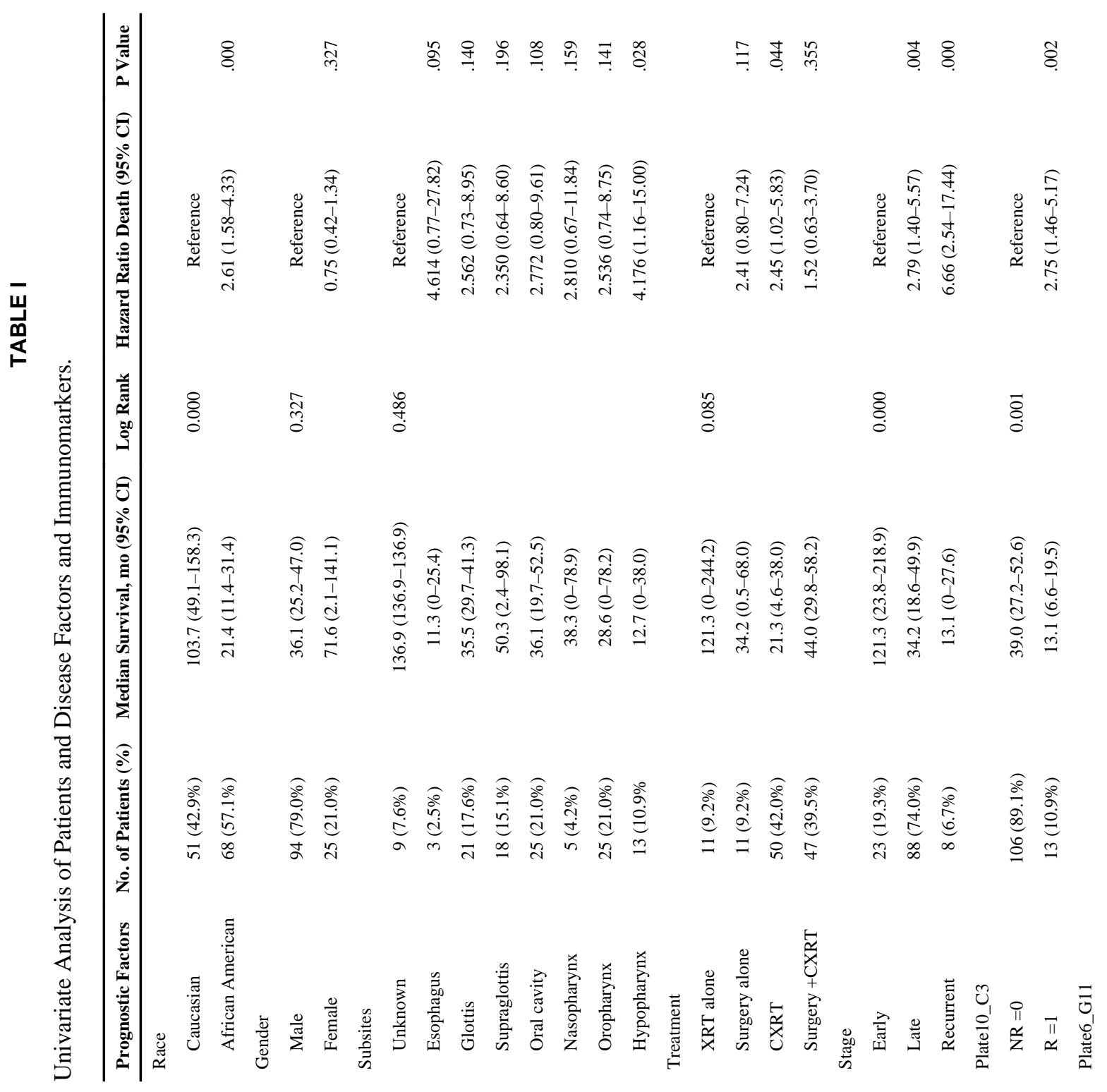




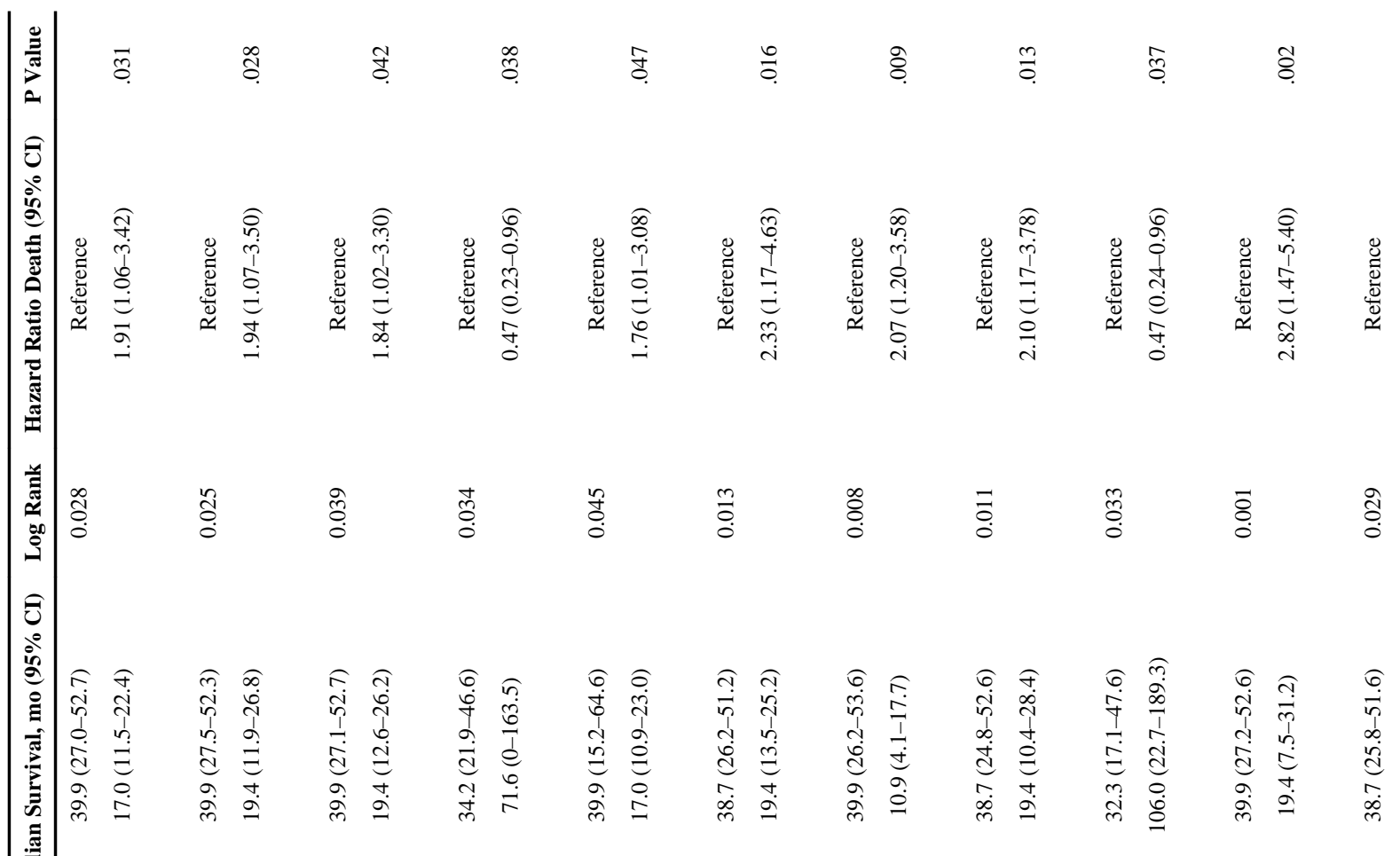

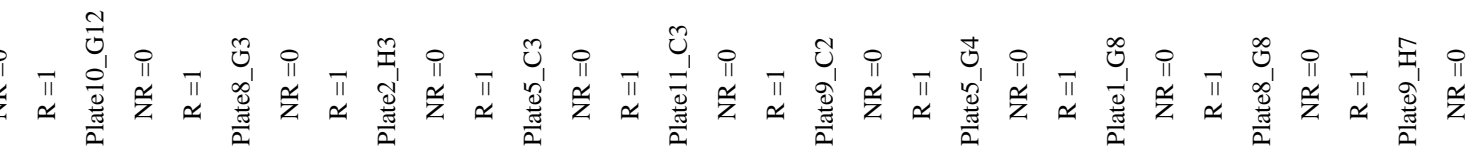




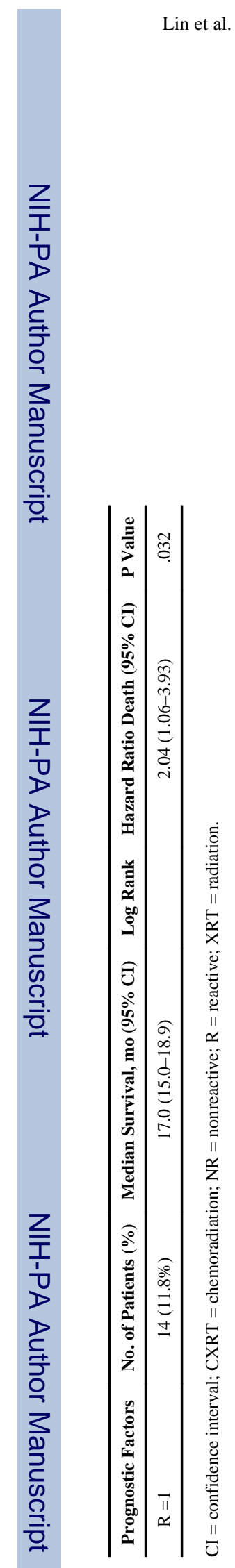


TABLE II

Multivariate Analysis.

\begin{tabular}{|c|c|c|}
\hline & Hazard Ratio for Death $(95 \%$ CI) & P Value \\
\hline 10_C3-reactive & $2.42(1.26-4.64)$ & .008 \\
\hline African American & $2.62(1.56-4.39)$ & .000 \\
\hline Late stage & $2.62(1.31-5.21)$ & .006 \\
\hline Recurrent stage & $4.75(1.79-12.60)$ & .002 \\
\hline 6_G11-reactive & $1.94(1.07-3.53)$ & .030 \\
\hline African American & $2.37(1.42-3.98)$ & .001 \\
\hline Late stage & $3.07(1.53-6.14)$ & .002 \\
\hline Recurrent stage & $5.93(2.26-15.54)$ & .000 \\
\hline 10_G12-reactive & $2.47(1.33-4.59)$ & .004 \\
\hline African American & $2.53(1.51-4.23)$ & .000 \\
\hline Late stage & $3.24(1.59-6.58)$ & .001 \\
\hline Recurrent stage & $5.99(2.25-15.95)$ & .000 \\
\hline 8_G3-reactive & $1.62(0.88-2.96)$ & .119 \\
\hline African American & $2.38(1.41-4.00)$ & .001 \\
\hline Late stage & $2.96(1.48-5.92)$ & .002 \\
\hline Recurrent stage & $5.53(2.11-14.51)$ & .001 \\
\hline 2_H3-reactive & $0.70(0.32-1.50)$ & .355 \\
\hline African American & $2.27(1.32-3.93)$ & .003 \\
\hline Late stage & $2.74(1.37-5.50)$ & .005 \\
\hline Recurrent stage & $5.80(2.19-15.31)$ & .000 \\
\hline 5_C3-reactive & $1.44(0.80-2.61)$ & .225 \\
\hline African American & $2.62(1.53-4.50)$ & .000 \\
\hline Late stage & $2.45(1.14-5.23)$ & .021 \\
\hline Recurrent stage & $5.18(1.66-16.17)$ & .005 \\
\hline 11_C3-reactive & $1.80(0.90-3.60)$ & .095 \\
\hline African American & $2.42(1.44-4.05)$ & .001 \\
\hline Late stage & $2.81(1.41-5.61)$ & .003 \\
\hline Recurrent stage & $5.40(2.05-14.24)$ & .001 \\
\hline 9_C2-reactive & $2.29(1.31-4.02)$ & .004 \\
\hline African American & $2.69(1.60-4.52)$ & .000 \\
\hline Late stage & $2.94(1.47-5.88)$ & .002 \\
\hline Recurrent stage & $5.25(1.99-13.83)$ & .001 \\
\hline 5_G4-reactive & $2.49(1.37-4.54)$ & .003 \\
\hline African American & $2.79(1.65-4.72)$ & .000 \\
\hline Late stage & $3.14(1.57-6.30)$ & .001 \\
\hline
\end{tabular}

Laryngoscope. Author manuscript; available in PMC 2015 August 01. 


\begin{tabular}{lcc}
\hline & Hazard Ratio for Death (95\% CI) & P Value \\
\hline Recurrent stage & $5.50(2.10-14.40)$ & .001 \\
\hline 1_G8-reactive & $0.55(0.26-1.13)$ & .103 \\
African American & $2.31(1.36-3.92)$ & .002 \\
Late stage & $3.03(1.51-6.12)$ & .002 \\
Recurrent stage & $5.87(2.21-15.55)$ & .000 \\
\hline 8_G8-reactive & $2.95(1.53-5.71)$ & .001 \\
African American & $2.58(1.54-4.33)$ & .000 \\
Late stage & $3.00(1.50-6.00)$ & .002 \\
Recurrent stage & $5.06(1.86-13.74)$ & .001 \\
\hline 9_H7-reactive & $1.54(0.78-3.04)$ & .213 \\
African American & $2.38(1.41-4.01)$ & .001 \\
Late stage & $2.91(1.46-5.83)$ & .003 \\
Recurrent stage & $5.45(2.07-14.35)$ & .001 \\
\hline
\end{tabular}

$\mathrm{CI}=$ confidence interval. 